\title{
Changes in Hospital Admissions and Mortality for Complications of Cirrhosis: Implications for Clinicians and Health Systems
}

\author{
Samuel B. Ho*, Michael E. Matheny ${ }^{\dagger}$, and Bernd E. Schnabl* \\ *Department of Medicine, VA San Diego Healthcare System and University of California, San Diego, CA, and ${ }^{\dagger}$ Geriatric Research Education \\ and Clinical Care Service, VA Tennessee Valley Healthcare System and Department of Biomedical Informatics, Biostatistics, and Medicine, \\ Vanderbilt University, Nashville, TN, USA
}

See "Complications Requiring Hospital Admission and Causes of In-Hospital Death over Time in Alcoholic and Nonalcoholic Cirrhosis Patients." by Hee Yeon Kim, et al. on page 95, Vol. 10. No. 1, 2016

The prevalence of chronic liver disease and the resulting cirrhosis is increasing in various countries around the world. In the United States, between approximately 1990 and 2010 we have observed an overall increase in the prevalence of chronic liver disease to $15 \%$ of the entire population, ${ }^{1}$ and with that a $43 \%$ increase in deaths due to cirrhosis and a 118\% increase in deaths due to hepatocellular carcinoma (HCC). ${ }^{2}$ With cirrhosis comes the increased risk of complications that require hospitalization, which are often prolonged, expensive, and have a high mortality. In this context, Kim and colleagues ${ }^{3}$ describe the complications requiring hospital admission and causes of death in 2,165 patients from five referral hospitals in South Korea for the years of 2002 and 2011. The study is notable for several major findings. First, despite the fact that hepatitis B is the most common cause of cirrhosis in Korea, the majority of patients hospitalized for cirrhotic complications had cirrhosis due to alcohol in 2002 (52\%), which decreased only slightly in 2011 (49\%). Second, the major causes of hospitalizations in the earlier time period were variceal bleeding followed by hepatic encephalopathy (HE), ascites, and hepatic failure; whereas in the later time period there was a relative reduction in $\mathrm{HE}$ and spontaneous bacterial peritonitis (SBP), with an increase in hepatocellular cancer and hepatorenal syndrome (HRS). Third, they observed little change in hospital mortality, 8.6\% in 2002 to $9.1 \%$ in 2011. Interestingly, the trend over time for cause of hospital death decreased for variceal bleeding, HE, and SBP, but increased for the more end stage causes of hepatic failure and HRS; changes that were most striking for patients with alcoholic cirrhosis. These findings are obviously limited by the known limitations of retrospective medical record data extraction, and more data related to Model for End-stage Liver Disease or ChildPugh scores, length of hospitalization, and readmissions would have been useful. The strength of the study is the relatively large number of patients from five referral centers that would be broadly representative of similar referral centers in countries with universal combined government and private insurance.

These findings have several important implications for clinicians and health systems in terms of care for patients with cirrhosis. The data illustrate the significant impact of alcohol use both as a cause of cirrhosis and risk factor for hospitalization. The significance of this cause of cirrhosis complications will continue to grow as increasingly effective antiviral therapies for hepatitis B and C are more widely used. Only small incremental improvements in the treatment of alcoholism have been described recently, and studies show that deficiencies in access to care and to recommended treatment exist. ${ }^{4}$ Clearly, a focus on delivering evidence based treatment programs for patients with alcohol use disorders should be a high priority for efforts to prevent cirrhotic complications. On a more positive note, the changing trends in the causes of hospital admission imply that there have been improvements in care for the treatable complications such as variceal bleeding, HE, and SBP, with a concomitant increase in the more end-stage complications of hepatic failure, HCC, and HRS. These changes are expected and are likely related to the advances in treatments aimed at the complications of cirrhosis, as well as general improvements in hospital intensive care over time. Between the two decades studied in this report, high quality evidence from randomized

Correspondence to: Samuel B. Ho

Department of Medicine, VA San Diego Healthcare System and University of California, 3350 La Jolla Village Drive, San Diego, CA 92161, USA

Tel: +1-858-552-8585 (ext 2631), Fax: +1-858-552-4327, E-mail: Samuel.Ho2@va.gov

pISSN 1976-2283 eISSN 2005-1212 http://dx.doi.org/10.5009/gnl15593

@ This is an Open Access article distributed under the terms of the Creative Commons Attribution Non-Commercial License (http://creativecommons.org/licenses/by-nc/4.0) which permits unrestricted non-commercial use, distribution, and reproduction in any medium, provided the original work is properly cited. 
trials of treatments for decompensated cirrhosis have resulted in several practice guidelines from professional societies ${ }^{5,6}$ and recommended quality of care checklists. ${ }^{7}$ Improvements in the quality of hospital care for patients with cirrhosis in the Unites States may be contributing to a decline in mortality from 2002 to 2010 among a national dataset of patients hospitalized with cirrhosis in the Unites States. ${ }^{8}$

Despite advances in the evidence base for treating cirrhotic complications, the adherence to guidelines and optimal treatment in actual practice is low, at least in the Unites States, ${ }^{9,10}$ thus limiting the potential overall decrease in hospital admissions and mortality that could be achieved. To date several small, single center studies have been reported showing the potential effectiveness of care coordination and health information technology interventions in general hospital settings that could increase adherence to cirrhosis care guidelines. Wigg et al. ${ }^{11}$ found that increased decision support, patient self-management, and information system use resulted in greater adherence to outpatient clinic visits and HCC screening, but did not reduce readmission rates. Johnson et al. ${ }^{12}$ found that requiring the use of checklists for patients admitted with variceal bleeding resulted in increased adherence to clinical guidelines and reduced in hospital readmissions. Morando et al. ${ }^{13}$ have shown that use of "day hospitals" to facilitate outpatient management of ascites and SBP prophylaxis resulted in reduced 30-day readmissions and 12-month mortality. Recently, Tapper et al. ${ }^{14}$ have shown in a pre-post design that use of electronic clinical decision support tools for improving antibiotic prophylaxis and HE treatment resulted in fewer readmissions. Overall these studies demonstrate the important principle that significant improvements in access and adherence to guidelines is achievable for patients with cirrhotic complications, but further work is needed to show that these can be widely sustained in a variety of systems and still impact important outcomes. Because of the high risk, high cost potential of cirrhosis, data tracking complications, hospitalizations, and mortality will continue to be important. Common sense and current evidence support efforts to link this data to interventions for adherence to quality measures for cirrhosis care in order to maximally impact hospital admissions and mortality.

\section{CONFLICTS OF INTEREST}

No potential conflict of interest relevant to this article was reported.

\section{REFERENCES}

1. Younossi ZM, Stepanova M, Afendy M, et al. Changes in the prevalence of the most common causes of chronic liver diseases in the United States from 1988 to 2008. Clin Gastroenterol Hepatol 2011;9:524-530.e1.

2. Murray CJ, Atkinson C, Bhalla K, et al. The state of US health, 1990-2010: burden of diseases, injuries, and risk factors. JAMA 2013;310:591-608.

3. Kim HY, Kim CW, Choi JY, et al. Complications requiring hospital admission and causes of in-hospital death over time in alcoholic and nonalcoholic cirrhosis patients. Gut Liver 2016;10:95-100.

4. Connor JP, Haber PS, Hall WD. Alcohol use disorders. Lancet. Epub 2015 Sep 3. http://dx.doi.org/10.1016/S0140-6736(15)00122-1.

5. Garcia-Tsao G, Lim JK; Members of Veterans Affairs Hepatitis C Resource Center Program. Management and treatment of patients with cirrhosis and portal hypertension: recommendations from the Department of Veterans Affairs Hepatitis C Resource Center Program and the National Hepatitis C Program. Am J Gastroenterol 2009;104:1802-1829.

6. 0'Shea RS, Dasarathy S, McCullough AJ; Practice Guideline Committee of the American Association for the Study of Liver Diseases; Practice Parameters Committee of the American College of Gastroenterology. Alcoholic liver disease. Hepatology 2010;51:307-328.

7. Kanwal F, Kramer J, Asch SM, et al. An explicit quality indicator set for measurement of quality of care in patients with cirrhosis. Clin Gastroenterol Hepatol 2010;8:709-717.

8. Schmidt ML, Barritt AS, Orman ES, Hayashi PH. Decreasing mortality among patients hospitalized with cirrhosis in the United States from 2002 through 2010. Gastroenterology 2015;148:967977.e2.

9. Kanwal F, Kramer JR, Buchanan P, et al. The quality of care provided to patients with cirrhosis and ascites in the Department of Veterans Affairs. Gastroenterology 2012;143:70-77.

10. Volk ML, Tocco RS, Bazick J, Rakoski MO, Lok AS. Hospital readmissions among patients with decompensated cirrhosis. Am J Gastroenterol 2012;107:247-252.

11. Wigg AJ, McCormick R, Wundke R, Woodman RJ. Efficacy of a chronic disease management model for patients with chronic liver failure. Clin Gastroenterol Hepatol 2013;11:850-858.e4.

12. Johnson EA, Spier BJ, Leff JA, Lucey MR, Said A. Optimising the care of patients with cirrhosis and gastrointestinal haemorrhage: a quality improvement study. Aliment Pharmacol Ther 2011;34:7682.

13. Morando F, Maresio G, Piano S, et al. How to improve care in outpatients with cirrhosis and ascites: a new model of care coordination by consultant hepatologists. J Hepatol 2013;59:257-264.

14. Tapper EB, Finkelstein D, Mittleman MA, Piatkowski G, Chang M, Lai M. A quality improvement initiative reduces 30-day rate of readmission for patients with cirrhosis. Clin Gastroenterol Hepatol. Epub 2015 Sep 25. http://dx.doi.org/10.1016/j.cgh.2015.08.041. 